\title{
Payoff-oriented quantization and application to power control
}

\author{
Chao Zhang*, Nizar Khalfet*, Samson Lasaulce*, Vineeth Varma ${ }^{\dagger}$ and Sophie Tarbouriech ${ }^{\ddagger}$ \\ *LSS, CNRS-CentraleSupelec-Univ. Paris Sud, Gif-sur-Yvette, France \\ ${ }^{\dagger}$ CRAN (Univ. Lorraine), Nancy, France \\ ‡ LAAS-CNRS, Univ. de Toulouse, CNRS, Toulouse, France
}

\begin{abstract}
In many resource allocation problems, optimal allocation strategies must be determined when only a quantized version of the relevant parameters are available, for instance, power allocation in wireless communications. The contribution of this work is threefold. First, the quantization problem is revisited and a framework which encompasses the classical problem of quantization is proposed. Instead of minimizing the distortion, the goal is to minimize the gap between the maximum of a general payoff function (which would be reached by knowing all parameters of the function) and what is effectively reached when only the quantized version of the parameters is available. Then, to determine such a quantizer, the well-known Lloyd-Max algorithm is generalized. At last, we show how this framework can be applied to the problem of power control in wireless communications; the obtained numerical results clearly show the potential of such a framework.
\end{abstract}

\section{INTRODUCTION}

The primary motivation for formulating the technical problem under consideration in this paper comes from resource allocation problems in wireless communications. For this type of problems, quite often, one has to deal with the following situation. A payoff, reward or utility function $f(x ; g)$ has to be maximized with respect to the vector $x$ but its parameters (which are represented by the vector $g$ ) are not perfectly known. In this paper, we restrict our attention to the case where what is available to maximize $f$ is a quantized version of the function parameters. Although it has not been addressed from the technical perspective proposed in the present paper, this scenario is well motivated by numerous papers in the literature of wireless communications (see e.g., [1] [2] [3]). For instance, it is fully relevant when a transmitter has to perform power allocation by exploiting a quantized version of the channel which is sent by the receiver (through a feedback mechanism); in this example, the receiver needs to quantize the channel gains or matrix to meet some constraints e.g., linked to the feedback channel capacity.

The problem we introduce in this paper is the design of the quantizer which produces the distorted vector of parameters which is effectively available to maximize the payoff function $f$; in the power allocation problem which has been mentioned previously, the receiver has to quantize the downlink channel and send it to the transmitter whose role is to perform power allocation based on the quantized channel sent by the receiver. More precisely, we want to minimize the impact of quantization noise on the optimality loss which occurs when using the quantized version of $g$ to maximize the payoff function $f$. Of course, this design is performed under a resource constraint which is the number of quantization bits. The design of a quantizer consists in finding a partition and the corresponding representatives. Indeed, the space in which the vector of parameters lies has to be partitioned into cells or regions. For any input $g$ which belongs to a given cell, the quantizer produces the same output $\hat{g}$, which is called the representative of the considered cell.The maximum number of cells the quantizer can use is given by the total number of bits available for quantizing.

To determine the best quantizer in the sense of minimizing the optimality loss induced by using $\widehat{g}$ instead of $g$, we generalize the well-known Lloyd-Max algorithm (LMA) [4] [5]. Indeed, the LMA-based quantizer aims at minimizing the distortion i.e., the mean square error between the source and its reconstructed version. But, note that this design is independent of the use of the quantized quantity. It turns out that the quantizer design might be improved when measured in terms of the final utility of payoff. The original version of the LMA has been generalized in many diverse ways. For instance, it has been generalized to scenarios where the source to be quantized has to be sent through a noisy channel (see e.g., [6] [7]) and the source is itself noisy [8]. However, almost always, the performance criterion is the distortion. There exist some other works where a different performance criterion is considered such as [9] where the $L_{p}-$ norm is considered (instead of the Euclidean norm) or some specific performance criterion such as in [10] where the goal is to obtain a quantized beamforming vector. More generally, in [11], the author considers the problem of minimizing an arbitrary function of the difference between the actual vector of parameters and its quantized version but, again, this problem does not correspond to the framework of payoff-oriented quantization we propose here. Recently, several works like [12] adrress the issue of quantization noise as a primary concern in resource allocation problems. As a result, some new studies like [13], optimize the allocation of quantization bits specifically for sum-rate maximization. However, in these works the goal is not to design the complete quantization scheme for their payoff (i.e., the allocation of bits, partitions and representatives). Finally, some papers study situations where specific control-theoretic performance criteria are optimized (see e.g., [14]) but the quantization problem is not stated in general and not solved 
by using a generalized version of the LMA. To the best knowledge of the authors, the quantization problem has not been formulated as in the present work.

The paper is structured as follows. In Sec. 2, the proposed quantization problem formulation is provided. In Sec. 3, a generalized version of the LMA is provided and in Sec. 4 some specific applications of our scheme in wireless resource allocation problems is discussed. Finally, in Sec. 5 we provide a numerical performance analysis.

\section{Problem Statement}

We consider a function $f(x ; g): \mathcal{X} \times \mathcal{G} \rightarrow \mathbb{R}$, referred to as the payoff function. Both $x \in \mathcal{X}$ and $g \in \mathcal{G}$ may be vectors in general with $X \subset \mathbb{R}^{N}$ and $\mathcal{G} \subset \mathbb{R}^{K}$. The ultimate goal is to maximize $f$ with respect to $x$ while only knowing a quantized version of $g$, which is denoted by $\mathcal{Q}(g)$. Here, the objective is to find a good quantizer namelely a quantizer which allows to mimimize the impact of quantization on the optimality loss induced by using $\mathcal{Q}(g)$ in the final optimization problem.

A quantizer $\mathcal{Q}$ is given by a partition of $\mathcal{G}$ into cells and their representatives. We denote by $M$ the maximum number of cells. The cells are denoted by $\left\{\mathcal{C}_{1}, \ldots, \mathcal{C}_{M}\right\}$ and verify $\mathcal{C}_{m} \subset$ $\mathcal{G}$ such that $\mathcal{C}_{1} \cup \mathcal{C}_{2} \cup \cdots \cup \mathcal{C}_{M}=\mathcal{G}$ and $\mathcal{C}_{m} \cap \mathcal{C}_{n}=\varnothing$ for any $m \neq n$. The quantization rule is assumed to be as follows: $\mathcal{Q}(g)=r_{m}$ if $g \in \mathcal{C}_{m}$ where $m=1,2, \ldots, M$. The conventional approach consists in determining $\mathcal{Q}$ so that the distortion is minimized i.e., to minimize $D=\int \phi(g) \| \mathcal{Q}(g)-$ $g \|^{2} \mathrm{~d} g$ where $\phi$ is the probability density function (p.d.f.) of $g$. The advantage of such an approach is that it may be possible to make explicit the quantizer (namely the representatives and cells) and this leads to a scheme which is independent of the payoff. However, if the payoff is known, it is generally possible to further improve the performance when it is measured in terms of final payoff. Indeed, if one denotes by $F$ the actual maximum of $f$

$$
F(g)=\max _{x} f(x ; g)
$$

and by $\widehat{F}$

$$
\widehat{F}(\mathcal{Q}(g))=\max _{x} f(x ; \mathcal{Q}(g))
$$

the level which is effectively attained by only knowing $\mathcal{Q}(g)$ and not $g$, it is relevant to determine $\mathcal{Q}$ through the following relation:

$$
\mathcal{Q}^{\star} \in \arg \min _{\mathcal{Q}} \mathbb{E}_{g}\|F(g)-\widehat{F}(\mathcal{Q}(g))\|^{2} .
$$

The purpose of the next section is precisely to provide results in order to minimize the quantity defined in (3). Just as in the LMA, in general there is no guarantee for global optimality. This classical issue is left as an extension of the present work, the goal being here to focus on what is really novel.

\section{GENERAL QUANTIZATION SCHEME}

When $g$ is a $K$-dimensional vector $(K>1)$, finding jointly the cells and the representatives which minimize (3) is a highly non-trivial problem. This is the main reason why we take inspiration from classical quantization schemes such as the LMA, in order to search for a scheme that can find the most suitable representatives $\left\{r_{1}, \ldots, r_{M}\right\}$ (locally optimal) for a given set of cells $\left\{\mathrm{C}_{1}, \ldots, \mathcal{C}_{M}\right\}$, and to find locally optimal cells for a given set of representatives. Once these can be found, we can iteratively solve for $\left\{r_{1}, \ldots, r_{M}\right\}$ and $\left\{\mathcal{C}_{1}, \ldots, \mathcal{C}_{M}\right\}$, to find a locally optimal solution (not necessarily globally optimal).

Finding the optimal cells for a given set of representatives $\left\{r_{1}, \ldots, r_{M}\right\}$, can be done in a manner similar to the classical LMA, and by constructing the partitions that are similar to Voronoi partitions:

$$
\mathcal{C}_{m}=\left\{g \in \mathbb{R}^{K}:\left[F(g)-F\left(r_{m}\right)\right]^{2} \leqslant\left[F(g)-F\left(r_{n}\right)\right]^{2}\right\}
$$

where $n \neq m$. Clearly, the motivation for this choice is that instead of the Euclidean distance or distortion of $G$ as taken in the classical Voronoi partition, here we look for the set with the minimum distortion in terms of $F(g)$. On the other hand, finding the optimal representative $r_{m}$ for a given cell $\mathcal{C}_{m}$, might not be as straightforward. Indeed, when $G$ is a scalar and $F$ is invertible, and if $\mathcal{C}_{m}$ is defined by an interval of the form $\left[t_{m}, t_{m+1}\right]$, then the best representative $r_{m}^{\star}$ is given by

$$
F\left(r_{m}^{\star}\right)=\frac{\int_{t_{m}}^{t_{m+1}} \phi(g) F(g) \mathrm{d} g}{\int_{t_{m}}^{t_{m+1}} \phi(g) \mathrm{d} g} .
$$

The above result can be shown to be true by differentiating $\mathbb{E}_{g}\left(\|F(\widehat{g})-F(g)\|^{2}\right)$ w.r.t $r_{m}$ in a given cell $\mathcal{C}_{m}=\left[t_{m}, t_{m+1}\right]$. Since $F$ is invertible, its derivative never vanishes and thus (5) can be obtained. Although $F$ is typically an invertible function when $g$ is scalar, this property is generally lost when $g$ becomes a vector and the $K$ elements of $r_{m}$ can not be recovered from only one equation (1 equation for $K$ unknowns). For this reason, when $g$ is the vector of the form $g=\left(g_{1}, g_{2}, \ldots, g_{K}\right)^{T}$, some assumptions have to be added to make the identification procedure possible. The next result is precisely based on one reasonable assumption which allows identifiability to be possible.

Assumption III.1 (Decomposability assumption). The function $F$ can be written as $F=\sum_{k=1}^{K} u_{k}$ with $u_{k}: \mathcal{G} \rightarrow$ $\mathbb{R}$ and it is such that then the vector function $V(g):=$ $\left(u_{1}(g), \ldots, u_{K}(g)\right)^{\mathrm{T}}$ is invertible in $\mathrm{C}_{m}$.

This assumption is well suited for several applications where the total payoff is the sum of several components, for example, when the payoff is the sum of the rates over each band. Indeed, the function $V$ may not be globally invertible, but our results can be applied to partitions $\mathcal{C}_{m}$ such that this assumption holds as we will illustrate in Sec. 4. For wireless systems, this sufficient condition is often met due to the monotonicity of most payoff functions; indeed, many payoff functions in wireless communications are typically monotonically increasing w.r.t. the signal-to-interference plus noise ratio (SINR). 
Proposition III.2 (Optimal representatives). If Assumption III.1 holds for a partition $\mathcal{C}_{m}$, with the decomposed invertible function being $V(g)=\left(u_{1}(g), \ldots, u_{K}(g)\right)^{\mathrm{T}}$, then the optimal representative $r_{m}^{*}$ which minimize $\mathbb{E}_{g}\left(\|V(\hat{g})-V(g)\|^{2}\right)$ also minimize $\mathbb{E}_{g}\left(\|F(\widehat{g})-F(g)\|^{2}\right)$ where $\hat{g}=\mathcal{Q}(g)$, and can be obtained by solving the following system of $K$ equations

$$
u_{k}\left(r_{m}^{*}\right)=\frac{\int_{\mathfrak{C}_{m}} \phi(G) u_{k}(G) \mathrm{d} G}{\int_{\mathfrak{C}_{m}} \phi(G) \mathrm{d} G}
$$

Proof. For a given quantization region $\mathrm{C}_{m}$, the gradient of $E_{g}\left(\|V(\hat{g})-V(g)\|^{2}\right)$ with respect to $r_{m}$ can be written as:

$$
\begin{aligned}
& \nabla E_{g}\left(\|V(\widehat{g})-V(g)\|^{2}\right) \\
= & \frac{\partial E_{g}\left(\|V(\widehat{g})-V(g)\|^{2}\right)}{\partial r_{m}} \\
= & \int_{\mathfrak{C}_{m}} \phi(g) \frac{\partial\left\|V\left(r_{m}\right)-V(g)\right\|^{2}}{\partial r_{m}} \mathrm{~d} g \\
= & \int_{\mathfrak{C}_{m}} \phi(g) J_{V}\left(r_{m}\right) \frac{\partial\left\|V\left(r_{m}\right)-V(g)\right\|^{2}}{\partial V\left(r_{m}\right)} \mathrm{d} g \\
= & \int_{\mathfrak{C}_{m}} 2 \phi(g) J_{V}\left(r_{m}\right)\left[V\left(r_{m}\right)-V(g)\right] \mathrm{d} g
\end{aligned}
$$

where $J_{V}$ is the Jacobian matrix of $V$ evaluated at $r_{m}$, i.e.,

$$
J_{V}\left(r_{m}\right)=\left[\begin{array}{ccc}
\frac{\partial u_{1}}{g_{1}}\left(r_{m}\right) & \ldots & \frac{\partial u_{1}}{g_{K}}\left(r_{m}\right) \\
\vdots & \vdots & \vdots \\
\frac{\partial u_{K}}{g_{1}}\left(r_{m}\right) & \ldots & \frac{\partial u_{K}}{g_{K}}\left(r_{m}\right)
\end{array}\right] .
$$

At the local minimum, we must have the gradient of $E_{g}\left(\|V(\widehat{G})-V(G)\|^{2}\right)$, become zero. Since, we assume that $V(\cdot)$ is invertible in $\mathcal{C}_{m}$, we can use the inverse function theorem to conclude that $J_{V}\left(L_{m}\right)$ is invertible at all points in $\mathcal{C}_{m}$. As a result, we have

$$
\int_{\mathcal{C}_{m}} \phi(g)\left[V\left(r_{m}\right)-V(g)\right] \mathrm{d} g=0 .
$$

Hence, the optimum representatives can be obtained as

$$
V\left(r_{m}\right)=\frac{\int_{\mathcal{C}_{m}} \phi(g) V(g) \mathrm{d} g}{\int_{\mathcal{C}_{m}} \phi(g) \mathrm{d} g}
$$

which is equivalent to

$$
u_{k}\left(r_{m}\right)=\frac{\int_{\mathcal{C}_{m}} \phi(g) u_{k}(g) \mathrm{d} g}{\int_{\mathfrak{C}_{m}} \phi(g) \mathrm{d} g}
$$

which must hold for all $k=1,2, \ldots, K$. Taking the sum of (11) with respect to $k$, we have

$$
\sum_{k=1}^{K} u_{k}\left(L_{m}\right)=\frac{\sum_{k=1}^{K} \int_{\mathfrak{C}_{m}} \phi(G) u_{k}(G) \mathrm{d} G}{\int_{\mathfrak{C}_{m}} \phi(G) \mathrm{d} G}
$$

Knowing $F^{\star}(G)=\sum_{k=1}^{K} u_{k}(G)$, the optimum representatives to minimize $E_{g}\left(\|V(\widehat{G})-V(G)\|^{2}\right)$ satisfy the following condition

$$
F^{\star}\left(L_{m}\right)=\frac{\int_{\mathcal{C}_{m}} \phi(G) F^{\star}(G) \mathrm{d} G}{\int_{\mathcal{C}_{m}} \phi(G) \mathrm{d} G}
$$

which is the expression to minimize the payoff gap $E_{g}\left(\left\|F^{\star}(\widehat{G})-F^{\star}(G)\right\|^{2}\right)$.

The scalar case (3) can be checked to obtained by setting $K$ to 1 . Now, we exploit Proposition III.2 to derive a suitable algorithm to find a payoff-oriented quantizer knowing $F$.

Inputs: $\phi(G): \mathbb{R}^{K} \rightarrow \mathbb{R}_{\geqslant 0}, F^{\star}=\sum_{k} u_{k}$ satisfying assumption III.1, $\left\{r_{1}^{(0)}, \ldots, r_{M}^{(0)}\right\}$

Outputs: $\left\{r_{1}^{\star}, \ldots, r_{M}^{\star}\right\},\left\{C_{1}^{\star}, \ldots, C_{M}^{\star}\right\}$

Initialization: Set iteration index $q=0$. Initialize the quantization representatives according to $\left\{r_{1}^{(0)}, \ldots, r_{M}^{(0)}\right\}$. Set $r_{m}^{(-1)}=0$ for all $m \in\{1, \ldots, M\}$.

while $\sum_{m=1}^{M}\left(r_{m}^{(q)}-r_{m}^{(q-1)}\right)^{2}>\delta$ and $q<Q$ do Update the iteration index: $q \leftarrow q+1$.

For all $m \in\{1,2, . ., M\}$, update $C_{m}^{q}$ from $r_{m}^{q-1}$ using (4).

For all $m \in\{1,2, . ., M\}$, update $r_{m}^{q}$ for each partition $C_{m}^{q}$ using (6).

end

$\forall m \in\{1, \ldots, M\}, r_{m}^{\star}=r_{m}^{(q)}, t_{m}^{\star}=t_{m}^{(q)}, t_{M+1}^{\star}=+\infty$

Algorithm 1: Algorithm to obtain the payoff-oriented quantizer

The new quantizer can be summarized by the following algorithm. It is also important to note that the LMA can be treated as the first order Taylor approximation of the proposed algorithm, or just a special case in which $F$ is a linear function. Note that Assumption III.1 may not hold over the entire set $\mathcal{G}$, in this case, $\mathcal{G}$ can be partitioned into several sub-regions where each region can be quantized specifically. For example, as seen in the next section, when energy maximization is pursued, solutions are such that only the best channel is picked in which case just the best channel needs to be quantized and then the minimum number of bits can be allocated to the other channels.

\section{APPLICATION TO TYPICAL WIRELESS PAYOFF FUNCTIONS}

\section{A. Energy-efficiency maximization}

In this subsection, we consider a particular payoff function, the energy efficiency function, for a multi-band scenario. The quantizers of interest for the transmitter to allocate its power are given by the channel gains. The channel gain in band $k$ is denoted by $g_{k}=\left|h_{k}\right|^{2}$ where $h_{k}$ may typically be the realization of a complex Gaussian random variable if Rayleigh fading is considered. The power emitted in band $k$ is denoted by $p_{k}$ and is assumed to be subject to power limitation as: 
$p_{k} \geqslant 0$ and $\sum_{k=1}^{K} p_{k} \leqslant P_{\max }$. The K-dimensional column vector formed by the transmit power levels and channel gains will be denoted by $p=\left(p_{1}, \ldots, p_{K}\right)^{\mathrm{T}}$ and $g=\left(g_{1}, \ldots, g_{K}\right)^{\mathrm{T}}$, respectively. Here we choose the efficiency function of [15], which is defined as

$$
f(p, g)=\frac{\sum_{k=1}^{K} e^{\left(-\frac{c \sigma^{2}}{g_{k} p_{k}}\right)}}{\sum_{k=1}^{K} p_{k}}
$$

where $\sigma^{2}$ is the receive noise variance and $c=2^{r}-1$ with $r$ being the spectral efficiency. To find $F$ in the case of energyefficiency maximization, we first derive the optimal power control policy. This is the purpose of the next proposition.

Proposition IV.1. In multi-band scenario, to maximize the system energy-efficiency of, the optimal power allocation scheme is

$$
p_{k}^{\star}\left(g_{k}\right)= \begin{cases}0 & k \neq \arg \max _{i} g_{i} \\ \min \left(\frac{c \sigma^{2}}{g_{k}}, P_{\max }\right) & k=\arg \max _{i} g_{i}\end{cases}
$$

Proof. The proof is omitted because of the lack of space. It can be done by applying the scalar version of Proposition IV.1 to quantize the selected channel $g_{k}$ and selectively picking $\mathcal{C}_{m}$ as subsets of $\left\{g_{k}\right\}$.

According to Proposition IV.1, to maximize energyefficiency, the transmitter will only transmit through the best channel. To better estimate the payoff at the transmitter side, we can use the vector quantization method proposed in the previous subsection. However, this will entail a high complexity if the number of bands is very large. Since only one band is active in each time-slot, this property can be used to design a special quantization scheme. Firstly, we divide the whole region into $K$ sub-regions $\widehat{\mathcal{C}}_{1}, \ldots, \widehat{\mathcal{C}}_{K}$, where $\widehat{\mathfrak{C}}_{k}=\left\{g \in \mathbb{R}^{K}: g_{k}=\max g_{i}\right\}$. The region $\widehat{\mathcal{C}}_{k}$ corresponds to the region in which $g_{k}^{i}$ is the best channel. Without loss of generality, we consider the quantization scheme for region $\widehat{C}_{k}$. If the channel realization belongs to $\widehat{C}_{k}$, then only band $k$ will be active. It implies that only selection of band $k$ and the value of $g_{k}$ are useful to improve the energy efficiency. Suppose $Q(g)=\left(\widehat{g}_{1}, \ldots, \widehat{g}_{K}\right)^{\mathrm{T}}$, the first issue can be easily solved by setting the largest element of the representatives in $\widehat{\mathcal{C}}_{k}$ as $\widehat{g}_{k}$, i.e. $\max Q\left(g \in \widehat{\mathcal{C}}_{k}\right)=\widehat{g}_{k}$. Note that the optimal energy efficiency function in $\widehat{\mathcal{C}}_{k}$ can be simplified as

$$
F\left(g \in \widehat{\mathfrak{C}}_{k}\right)=\max _{p} f\left(p, g \in \widehat{\mathfrak{C}}_{k}\right)=\frac{e^{\left(-\frac{c \sigma^{2}}{g_{k} p_{k}^{\star}}\right)}}{p_{k}^{\star}\left(g_{k}\right)} .
$$

Hence, the second issue, to find the optimal quantized value of $\hat{g}_{k}$, can be solved by minimizing $d\left(\widehat{g}_{k}\right)$ defined as

$$
d\left(\widehat{g}_{k}\right)=\mathbb{E}_{g_{k} \mid g_{k} \geqslant g_{1}, \ldots, g_{k} \geqslant g_{K}}\left[F\left(g_{k}\right)-F\left(\widehat{g}_{k}\right)\right]^{2} .
$$

Proposition IV.2. Define the M-level scalar quantizer of $g_{k}$ transition levels set as $\left\{t_{k, 1}, \ldots, t_{k, M+1}\right\}$ and its corresponding representatives set as $\left\{r_{k, 1}, \ldots, r_{k, M}\right\}$. Suppose each channel $g_{k}$ is i.i.d. with p.d.f. $\phi\left(g_{k}\right)=\gamma e^{-\gamma g_{k}}$. Assume $P_{\max }$ is sufficiently large, for fixed representatives $\left\{r_{k, m}\right\}$, the intervals (cells) which minimize $d\left(\widehat{g}_{k}\right)$ can be obtained by

$$
t_{k, m}=\frac{r_{k, m-1}+r_{k, m}}{2}
$$

with fixed transition levels $\left\{t_{k, m}\right\}$, the optimum representatives to minimize $d\left(\widehat{g}_{k}\right)$ can be obtained by

$$
r_{k, m}=\frac{\int_{t_{k, m}}^{t_{k, m+1}} g\left(1-e^{-\gamma g}\right)^{K-1} \gamma e^{-\gamma g} \mathrm{~d} g}{\int_{t_{k, m}}^{t_{k, m+1}}\left(1-e^{-\gamma g}\right)^{K-1} \gamma e^{-\gamma g} \mathrm{~d} g} .
$$

Proof. The proof is omitted because of the lack of space.

Obtaining this scalar quantizer, $d\left(\widehat{g}_{k}\right)$ can be minimized knowing $g_{k}$ is the best channel. Without loss of generality, we assume that each region $\widehat{C}_{k}$ will be divided to $\mathrm{M}$ different quantization cells $\left\{\mathfrak{C}_{k, 1}, \ldots, \mathcal{C}_{k, M}\right\}$. The corresponding representative of the quantization region $\mathcal{C}_{k, m}$ is defined as a $\mathrm{K}$ dimensional vector $r_{k, m}=\left(r_{k, m}^{1}, \ldots, r_{k, m}^{K}\right)^{\mathrm{T}}$. Based on the results in the previous section, the vector quantization region $\mathcal{C}_{k, m}$ can be expressed as

$$
\mathcal{C}_{k, m}=\left\{g \in \mathbb{R}^{K}: t_{k, m}<g_{k} \leqslant t_{k, m+1}\right\} \cap \widehat{\mathcal{C}}_{k} .
$$

The corresponding representative $L_{k, m}$ can be chosen as:

$$
\begin{gathered}
r_{k, m}^{k}=r_{k, m} \\
r_{k, m}^{k^{\prime}}=\text { const }<r_{k, m}^{k}\left(k^{\prime} \neq k\right) .
\end{gathered}
$$

For the vector quantization of $g$, the quantization region set $\mathcal{C}=\left\{\mathrm{C}_{1,1}, \mathrm{C}_{1,2}, \ldots, \mathrm{C}_{K, M}\right\}$ and the representatives set $r=$ $\left\{r_{1,1}, r_{1,2}, \ldots, r_{K, M}\right\}$ can be found using (20)(21)(22). With this new approach, the complexity of the computation has been considerably reduced.

\section{B. Spectral efficiency maximization}

Using the same notations as in the previous section, here we consider the following well-known sum-rate function

$$
u^{\text {sum-rate }}(p, g)=\sum_{k=1}^{K} \log \left(1+\operatorname{SINR}_{k}(p, g)\right)
$$

where $\operatorname{SINR}_{k}(p, g)=\frac{g_{k} p_{k}}{\sigma^{2}}$. The optimum power allocation policy is given by the water-filling solution, i.e.,

$$
p_{k}^{\star}=\left[\mu-\frac{\sigma^{2}}{g_{k}}\right]^{+}
$$

where the water level $\mu$ can be obtained by solving $\sum_{k=1}^{K} p_{k}=$ $P_{\max }$ and the function $[x]^{+}=\max (x, 0)$. However, due to the incertitude of the function $[x]^{+}$, it is difficult to obtain the explicit expression for the function $F^{\star}(G)$. To express $F^{\star}(G)$ 
explicitly, we can firstly divide the whole region to specific partitions for each case corresponding to if $p_{k}^{*}=0$ or not. In each of these partitions, a different quantization scheme must be ideally used for optimal results as we cannot find $u_{k}$ satisfying Assumption III.1 for the general channel space. So, we focus on a practically relevant case of high signal-to-noise ratio (SNR) for the purpose of this work. Studying the general case is left as an extension which can be solved by treating each partition (corresponding to $p_{k}^{*}=0$ or not) separately.

In the high SNR case, i.e., when $\frac{P_{\max }}{\sigma^{2}} \rightarrow \infty$ we have $p_{k}^{*}>0$ for all $k$. Further, it can be observed that the sum-rate can be decomposed to individual payoff. Substituting (24) into (23), the individual payoff can be expressed as follows:

$$
\begin{aligned}
u_{k}^{\text {sum-rate }} & =\log \left(1+\mathrm{SINR}_{k}\right) \\
& =\log \left(1+g_{k} \mathrm{SNR}+\sum_{j \neq k} \frac{g_{k}}{g_{j}}\right)-\log (2)
\end{aligned}
$$

with SNR $:=\frac{P_{\max }}{\sigma^{2}}$. This $V=\left(u_{1}, u_{2}, \ldots, u_{K}\right)^{T}$ is clearly invertible w.r.t $g$ satisfying Assumption III.1, and we can therefore directly apply proposition III.2 and algorithm 1 to obtain the payoff oriented quantizer.

\section{NUMERICAL PERFORMANCE ANALYSIS}

In this section, we present simulation results to illustrate the performance of the proposed quantizer for a single user multi-band scenario. For comparison with the classical LMA, we look at the optimality loss induced by quantization defined as

$$
\Delta F(\%)=\mathbb{E}_{G}\left[\left|\frac{F(G)-\hat{F}(\mathcal{Q}(G))}{F(G)}\right|\right] \times 100
$$

which we term the relative optimality loss, where expectation is calculated by Monte-Carlo simulations for the channel gain $G$. For all $k$, the channel gain $g_{k}$ in band $k$ is assumed to be exponentially distributed, namely, its p.d.f. writes $\phi\left(g_{k}\right)=\exp \left(-g_{k}\right)$; this corresponds to the well known standard Rayleigh fading assumption. The considered scenario for all simulations is such that the transmit power $P_{\max }=$ $20 \mathrm{dBm}$ and a normalized receive noise power $\sigma^{2}=0 \mathrm{dBm}$, resulting in $\mathrm{SNR}=20 \mathrm{~dB}$.

In Fig. 1, the energy-efficiency payoff function defined in (14) is considered as the payoff function $f$ and the relative optimality loss is plotted against the number of quantization bits when the number of bands $K=16$. Our quantizer is obtained by using the proposition IV.1 we have provided.

In Fig. 2 we look the at the sum-rate payoff function as defined in (23). There are two bands available for communication, i.e. $K=2$. We plot the relative optimality loss induced by quantization w.r.t the number of quantization bits used.

As expected, when the number of bits increases, the relative optimality loss of both quantizers decreases. The proposed quantizer in Sec. 4 outperforms the classical LM vector quantizer. It is also important to note that the relative optimality loss of our quantizer is very close to 0 when we have more than 7 quantization bits, but the relative optimality

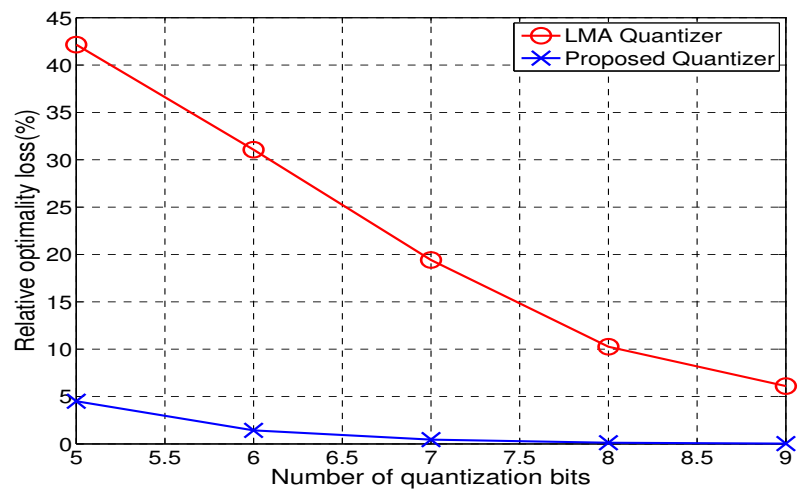

Fig. 1. Comparison of the performance in terms of final payoff between the conventional paradigm-based quantizer (which aims at minimizing distortion) and the proposed payoff-oriented quantizer. The figure represents the relative optimality energy-efficiency loss against number of quantization bits. The proposed quantizer results in a relative optimality loss (w.r.t. the case where the channel is known perfectly to the transmitter) of just $5 \%$ with 5 quantization bits compared to over $40 \%$ when using the classical.

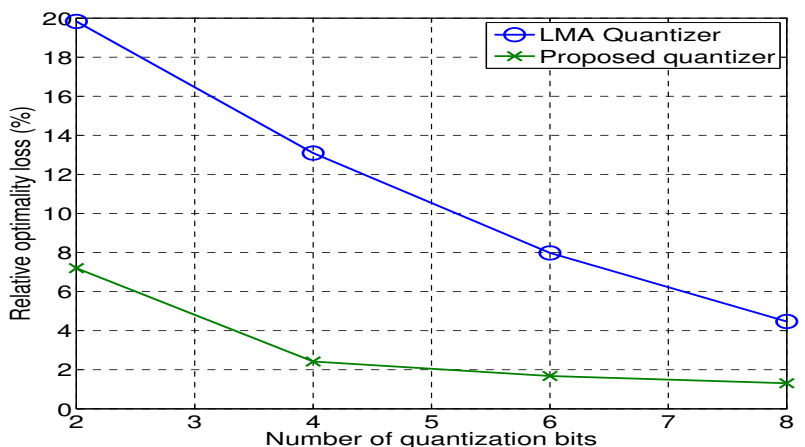

Fig. 2. Relative optimality spectral efficiency loss (sum-rate) based payoff against number of quantization bits. The proposed quantizer achieves a better performance and the loss is less than $5 \%$ with more than 5 quantization bits.

loss of LM quantizer still remains significant even with 9 quantization bits. Meanwhile, it can be predicted the novel quantizer will have the same performance as LM quantizer when the number of quantization bits tends to infinity since the relative optimality loss tends to 0 for both quantizer.

Finally, we study the relative optimality loss as a function of the number of bands with a fixed number of quantization bits. The number of quantization bits is set to five for this simulation. Fig. 3 illustrates the relative optimality loss against different number of bands. In single band scenario, the proposed quantizer coincides with the LM quantizer as minimizing the relative optimality loss is identical to minimization of distortion. In the multi-band case however, our quantizer achieve a better performance in terms of relative optimality loss and the difference becomes more significant as number of bands increases. Since the number of quantization bits is fixed, the accuracy of each component $\widehat{g}_{k}$ will degrade as the number of bands increases for the LMA. 


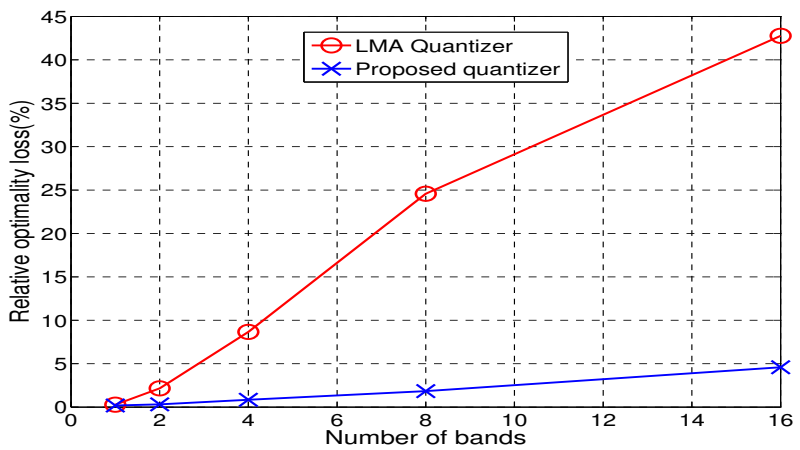

Fig. 3. Relative optimality energy-efficiency loss against number of bands. The proposed quantizer improves the performance in multi-band scenario and the improvement becomes more significant as the number of bands increases.

\section{CONCLUSION}

In this paper, the classical problem of quantization is revisited. Instead of considering the distortion or minimum mean square error to design the quantizer, the final use of the quantized parameters is considered. This approach is fully relevant in problems such as power control since the transmitter has often only access to an estimate or quantized version of the parameters (e.g., the channel gains). To effectively determine a good payoff-oriented quantizer in the vector case, we make some sufficient but reasonable sufficient conditions on the payoff function (such as the decomposability assumption) and resort to a suboptimal iterative algorithm. The benefit from implementing the proposed payoff-oriented quantization approach is illustrated with the problem of energy-efficient and spectral efficient power control problem. Significant gains can be obtained in terms of payoff especially when the number of bits decreases. Extending the proposed iterative algorithm to obtain the global optimum solution for a given class of payoff functions constitutes a challenging but very important extension.

\section{REFERENCES}

[1] G. Caire, N. Jindal, M. Kobayashi and N. Ravindran, "Multiuser MIMO achievable rates with downlink training and channel state feedback." IEEE Transactions on Information Theory, 56(6), 2845-2866, 2010.

[2] M. Kontouris, R. De Francisco, D. Gesbert, D. Slock and T. Salzer, "Efficient metrics for scheduling in MIMO broadcast channels with limited feedback." 2007 IEEE International Conference on Acoustics, Speech and Signal Processing-ICASSP'07. Vol. 3. IEEE, 2007.

[3] V. Varma, S. Lasaulce and C. Zhang, "Power modulation: Application to inter-cell interference coordination." 2015 23rd European Signal Processing Conference (EUSIPCO), IEEE, 2015.

[4] S. Lloyd, "Least squares quantization in PCM." IEEE transactions on information theory, 28(2), 129-137, 1982.

[5] J. Max, "Quantizing for minimum distortion." IRE Transactions on Information Theory, 6(1), 7-12, 1960.

[6] A. Kurtenbach and P. Wintz. "Quantizing for noisy channels." IEEE Transactions on Communication Technology, 17(2), 291-302, 1969.

[7] N. Farvardin, "A study of vector quantization for noisy channels." IEEE Transactions on Information Theory, 36(4), 799-809, 1990.

[8] B. Djeumou, S. Lasaulce and A. G. Klein. "Practical quantize-andforward schemes for the frequency division relay channel," EURASIP Journal on Wireless Communications and Networking, 2007:2, Oct. 2007.
[9] L. Wang, N. Piotto and D. Schonfeld. "Boosting quantization for L p norm distortion measure." 2012 IEEE Statistical Signal Processing Workshop (SSP), IEEE, 2012.

[10] C. R. Murthy and B. D. Rao. "A vector quantization based approach for equal gain transmission." IEEE Global Telecommunications Conference, 2005.

[11] J. G. Proakis, Digital Communications, 4th ed. New York: McGrawHill, Inc., 2001.

[12] A. D. Dabbagh and D. J. Love, "Multiple antenna MMSE based downlink precoding with quantized feedback or channel mismatch", IEEE Transactions on communications, 56(11), 2008.

[13] R. Bhagavatula and R. W. Heath, "Adaptive limited feedback for sumrate maximizing beamforming in cooperative multicell systems", IEEE Transactions on Signal Processing, 59(2), 800-811, 2011.

[14] F. Ceragioli and C. De Persis. "Discontinuous stabilization of nonlinear systems: Quantized and switching controls." Systems and Control Letters, 56(7), 461-473, 2007.

[15] E. V. Belmega and S. Lasaulce, "Energy-efficient precoding for multipleantenna terminals." IEEE. Transactions on Signal Processing, 59(1), 329$340,2011$. 